\title{
COMETS AND THE COSMOGONY OF THE SOLAR SYSTEM
}

\author{
S. K. VSEKHSVYATSKY
}

\begin{abstract}
The quantitative processes of eruptive development of planets into comets and other small bodies is studied from the Physical and orbital evolution of these minor bodies. The escape of comets (and of their products of decay) into interstellar space has been of the order of $10^{30}$ to $10^{31} \mathrm{~g}$ during the 1 ifetime of the solar system. The mean density of the planets and of their large satellites, and their specific rotational energy, serve as independent checks of the amount of lost material. As far back as 1955 and 1962, the eruption theory has predicted the high volcanic activity on Venus and Mars and on some satellites, that was actually discovered by Mariner 10 and Venus 9-10. Interstellar molecules confirm that ejection of cometary gases is widespread in the Galaxy. Six new bright short-period comets discovered in 1975 provide direct evidence for comet formation in the system of Jupiter in its 1961-1969 period of high activity. Brought together, these facts prove that planetary bodies began their existence at stellar temperatures. They cooled down from the surface, forming crusts of rocky and icy materials, that have initiated a long period of eruptive evolution, characterized by numerous cataclysms with the ejection of tremendous amounts of gas and dust, separated by more quiescent phases, like the present state of the earth. Comets, asteroids, meteoritic and meteoric material bear witness for the explosive processes on planetary bodies.
\end{abstract}

\section{INTRODUCTION}

Neither Descartes', Kant's or Laplace's hypotheses, nor the numerous more recent modifications of the accretion theory, have been able to explain the main peculiarities of the solar system. In most cases, cosmologists have based their hypotheses on superficial analogies. For instance, Laplace saw in Herschell's planetary nebulae, a prototype for his hypothesis of the rotating protosun with separating rings. In the same way, Urey, Kuiper, Whipple et al. have tried to identify the dark globules with objects connected to the origin of the solar system. But new facts of observation have constantly disproved these analogies. Planetary nebulae (like the ring-like nebula in Lyra) turned out to be expelled shells, expanding at velocities of tens of kilometers per second. The Crab Nebula in Taurus, is the rapidly expanding debris of the supernova of 1054. As Van den Bergh (1972) points out, neither diffuse nebulae nor dark globules show any evidence for star birth or condensation of planetary systems.

Since visual analogies have carried theorists away on wrong tracks, the present author believes that the only possible approach to solar system cosmogony, 
lies in the investigation of those processes that have transformed and still transform the solar system. These processes can be studied by comparing the peculiarities of the different types of planetary bodies.

of those, the various classes of small bodies, namely the comets, asteroids, meteorites and meteoric material, bring the most important information. Many years ago, the present author (Vsekhsvyatsky 1933, 1934, 1950) has shown that the existence of Jupiter's, Saturn's, Uranus' and Neptune's families of shortperiod comets, as well as their rapid disintegration, demonstrates the permanent formation of cometary objects inside the solar system. The peculiarities of cometary orbits indicate that comets are still being born within the system of giant planets. Lagrange (1814) Proctor (1884) and Crommelin (1910) had already assumed that the giant planets were the source of comets. This assumption received a decisive support when the high rate of decay of comets was established (Vsekhsvyatsky 1930, 1933). Cometary ejections from the planets, or planetary explosions were also assumed later by Zavaritsky, Fesenkov (1952) Orlov, Lodochnikov, Putilin et al.

Unquestionable facts support this basic assumption, namely:

1. the separation of cometary families from the different giant planets;

2. the close approach of new short-period comets to Jupiter's system just prior to discovery;

3. the existence of ices in the comet's nuclei;

4. the irregular shape of asteroids;

5. the chemistry and structure of meteorites;

6. the display of activity on the surfaces of planets and satellites;

7. the existence of Saturn and Uranus rings.

The amount of matter ejected from the solar system does not contradict this assumption. The sun loses about $10^{20} \mathrm{~g} /$ year in the solar wind. At least $10^{20} \mathrm{~g}$ /year is also lost by those comets (30\% to $50 \%$ ) whose orbits are transformed into hyperbolic trajectories by the cumulative effect of small perturbations (van Woerkom, verified by numerical experiments on computers by Everhard 1969). The loss of cometary gas and dust thrown away in hyperbolic orbits, as observed in cometary tails, is also of the same order of magnitude.

\section{NEW F $/$ CTS OF COMETARY ASTRONOMY}

Recently, Lyttleton (1974) confirmed our conclusion of 1954: the Oort's hypothetical cloud of comets cannot exist. Therefore the short-period comets cannot be captured from a non-existent source of hypothetical "new" long-period comets in the Oort's sense. Lately, new short-period comets have appeared in large numbers: 7 in 1948-51, 5 in 1963-65, 8 in 1973-75. They are all young, as shown by their orbits (close approach to Jupiter before discovery) and physical peculiarities (tails and high brightness). The total outflow of matter from the solar system, from the rate of cometary ejection on quasi-parabolic orbits, extrapolated to the age of the solar system, is $10^{29}-10^{30} \mathrm{~g}$, which nearly corresponds to the total present mass of the planets.

These giant losses are displayed by activity processes on planetary surfaces and by the existence of unstable "young" asteroids that are the remnants of short-period comets after decay of their volatile fractions. The set of small planets is also in good agreement with conclusions from cometary studies (Vsekhsvyatsky 1955, 1956), and some meteoritic material also comes from a planetary crust.

The history of observations of planetary surfaces shows activity on Mercury, the Moon and Mars, ard the peculiar conditions on Venus can be explained only by a recent giant cataclysm on the planet. The grandiose events on Jupiter, noticed long ago, have come into a particularly active phase since 1961. A giant flare, ejection of dark (apparently ashen) material was followed by a decline of the planetary brightness. Jupiter's satellites have shown traces of rapidly 
dissipating atmospheres (Kuiper 1949). Around Jupiter, there must exist (Vsekhsvyatsky 1962) a ring of comets and meteorites ejected from the satellite surfaces; perturbations of this ring eject individual comets into the inner solar system.

The ring system of Saturn, and that (recently discovered) of Uranus both demonstrate the recent existence of powerful eruptive processes on these planets, for a ring is quickly destroyed by self-collisions and by satellite perburbations. The existence of an atmosphere on Titan and on Triton (Kuiper 1947) proves the existence of inner heat sources, implying volcanic activities. Halley's comet could be one of the members of a large family of Neptune's comets, that may have existed for no more than 2500 or 3000 years.

\section{THE MEAN DENSITY OF THE PLANETS}

The large density range of the planets may come from a differential ejection rate of their less dense surface layers, during their whole existence. The processes are almost finished for the terrestrial planets, and are still going on for the giant planets. In Fig. 1, the losses have been calculated on the

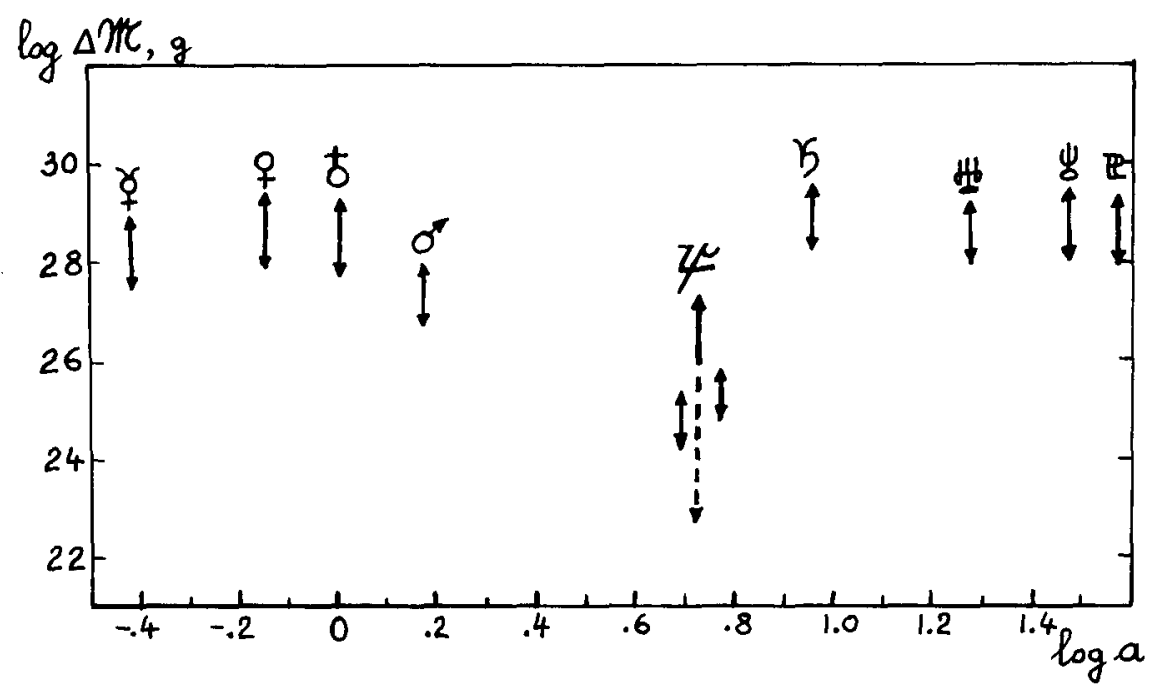

assumption of accepted density distribution laws for the Earth and other planets (Vseksvyatsky 1975). The loss of planetary material is $10^{28}-10^{30} \mathrm{~g}$, that is, the same order of magnitude as the total amount of cometary meteoritic matter ejected by the solar system. The energy required for ejecting $10^{27}-10^{28} \mathrm{~g}$ from a single planet like Venus is about $10^{40}$ ergs, which is compatible with the heat energy of protoplanets made of fragments of stellar material.

\section{CONDENSATION MODELS DO NOT FIT THE FACTS}

Condensation models have recently been more fashionable than eruptive models; see for instance Origin of the Solar System, Nice Colloquium (1972); however the numerous contradictory models confronted at Nice show that the question of whether the sun and the planets were formed simultaneously or by different processes, has not yet even been clarified. The eruptive evolution has however been developed by Dauvillier (1954), Woolfston (1969) and mainly by the present author (Vseksvyatsky 1933-1971). The high volcanic activity predicted by the 
author (1962) on Venus and Mars has been completely confirmed recently by the Soviet and American space probes Venera 4-8 and Mariner 9-10.

In his posthumous work, Kuiper (1974) tries to use the chemistry and mean density of the planets to suggest that the planetary system evolved from a quasi-double star, where the second star did not form, but condensed into a solar nebula. However, the whole process cannot be explained by the laws of dynamics. If the sun condenses, no external force could result in the formation of the solar nebula. This was obvious in our calculations of the 1950'ies, and shown again recently by Lyttleton (1974) who proved that the Oort's cloud did not exist.

\section{COMETARY MATERIAL IN THE INTERSTELLAR SPACE}

The total mass loss of the solar system material being $10^{30}$ to $10^{31} \mathrm{~g}$, the energy needed for its ejection is at least $10^{42}$ to $10^{43}$ ergs. This implies an energy supply at least two orders of magnitude larger than available from radioactive decay, rotational energy, and gravitational contraction. Therefore, the protoplanetary material was necessarily at a stellar temperature of the order of $10^{6} \mathrm{~K}$ or higher.

This conclusion that protoplanets were fragments of primary stellar bodies is consistent with our present knowledge; despite the billions of years of planetary cooling and eruptive evolution, Jupiter, Saturn, Uranus, Neptune, Venus, Titan, Triton and others still show indications of a high activity. This idea may be extended to the other stellar systems in the galaxy and the observed bright and dark nebulae, interpreted as the results of the same processes. According to Struve (1962), the total amount of diffuse material in the galaxy is $10^{8}$ to $10^{9} \mathrm{M}_{0}$; this approximately corresponds to the mass lost by the solar system $\left(10^{-3}\right.$ to $\left.10^{-2} \mathrm{M}_{\odot}\right)$. The long list of organic molecules recently detected by radio astronomy in the region of diffuse nebulae, and the striking analogy with the list of the cometary molecules, radicals and ions, suggests a similarity in the processes in the solar system and in the galactic nebulae. These clusters of complicated molecules of hydrocarbon and nitrogen groups of amino-acid type would be expected to form in the atmospheres of cooling stars and protoplanets, and to be expelled later in shells or through planetary eruptions. The outflow of matter indicated by the structure of the dark nebula supports these conclusions.

\section{EVOLUTION OF THE PROTOPLANETS AND FORMATION OF DIFFUSE MATTER IN THE GALAXY}

The specific rotational energy of the sun is two orders of magnitude smaller than that of Jupiter and Saturn. The missing energy (about $10^{44}$ ergs) has possibly been used for the formation of the solar system (Vsekhsvyatsky 1971) in accordance with the fragmentation scheme of a larger stellar body into the sun and the protoplanets.

Different in masses, but possessing nearly the same specific rotational energy, the primary protoplanets should have evolved independently because at a considerable distance from the sun. After rapidly passing through a stellarlike stage, the surfaces of protoplanetary bodies of smaller sizes should have cooled earlier, with the appearance of complex molecules in the outer layers, the condensation of mineral dust, and finally, (for the planets remote from the sun) the formation of snowy or icy shells by condensation of the atmosphere. This leads to the emprisonment of inner energy, resulting eventually in the explosive and volcanic processes that have ejected ices and fragments of crust into the interplanetary space.

Even now, the velocity of a volcanic gas saturated by ash can exceed $8 \mathrm{~km} /$ $\mathrm{sec}$, as observed at the eruption of the Krakatoa. Protoplanets with masses of the modern satellites (escape velocities of 1 to $3 \mathrm{~km} / \mathrm{sec}$ ) should have been 
the first to eject cometarymeteoroic material outside the solar system; some of them, exploding as a whole after their differentiation by specific weight, can explain the origin of the iron meteorites.

The driving force of eruptions appears to be the active gas freed by those chemical reactions proceeding inside the planets. Natural gas and oil on the earth are the consequence of these inner processes. Here Venus and the Saturn system are of a particular importance.

The present high atmospheric pressure and temperature of Venus indicate a recent burst of eruptive activity on the planets. The "greenhouse hypothesis" was disproved (Vsekhsvyatsky 1970) even before the Mariner 10 results. On the Venusian surface, there should exist seas of incandescent lava cooling off slow$1 y$ under the protection of a dense atmosphere. Saturn's rings (as we11 as the recently discovered ring system around Uranus) are unstable, therefore recent formations. The transformation of the cometary-asteroid cloud, formed by eruptions from the nearest satellites, into the present thin rings, must still be investigated, although it is clear that the eruptions in the Saturn system took place much earlier than the events on Venus.

\section{CONCLUSION}

The scales of the processes of the eruptive evolution of planets lead to the conclusion that all diffuse matter in the galaxy is a product of the activity of cooling stars and of protoplanets. A study of the radial velocities at radio frequencies should be undertaken to establish that interstellar molecules do not collapse towards centers, but are ejected from them. This would be a decisive confirmation of the eruption theory.

\section{REFERENCES}

Dauvillier, A. 1954, Mem. Soc. Roy. Sci. Liège, 4e Series, 14, 506.

Everhart, E. 1969, Astron. J., 74, 735.

Fesenkov, V. G. 1952, Voprosy kosmogonii. Akad. Nauk SSSR, N 1, P. 129. Highlights of Astronomy, 1971, 2, 193, ed. B. Middlehurst.

Kuiper, G. 1949, Atmospheres of the Earth and Planets, University of Chicago Press, Chicago.

Kuiper, G. 1974, Celestial Mechanics, 9, 321 .

Lyttleton, R. 1974, Astrophysics and Space Science, 27, N 2.

Novye idei $v$ astronomii. Komety, ikh priroda $i$ proiskhozhdenie. 1914, SintPetersburg.

Origin of the Solar System. 1972, Nice Symposium, Ed. H. Reeves, CNRS, Paris. Struve, 0., and Zebergs, V. 1962, Astronomy of the 20th Century, New York. Van den Bergh, S. 1972, Vistas in Astronomy, N 13.

Vsekhsvyatskij, S. K. 1930, Mon. Not. R.A.S., 40, 706.

Vsekhsvyastkij, S. K. 1933, Astron. Zh., 10, 29.

Vsekhsvyatskij, S. K. 1934, Astron. Zh., 11, 68; 437.

Vsekhsvyatskij, S. K. 1950, Astron. 2h., 27, 16.

Vsekhsvyatskij, S. K. 1951, Trudy i Soveshchaniya po Voprosam Kosmogonii, Moscow.

Vsekhsvyatskij, S. K. 1955, Astron. Zh., 32, 432.

Vsekhsvyatskij, S. K. 1956, Bul1. VAGO, N19, 66.

Vsekhsvyatskij, S. K. 1962, Astron. Zh., 39, 290.

Vsekhsvyatskij, S. K. 1962, Publ. Astr. Soc. Pacific, April, 74, 106.

Vsekhavyatskij, S. K. 1967, Priroda i proiskhozhdenie komet $i$ meteorov, Prosveshchenie, Moscow.

Vsekhavyatskij, S. K. 1970, Probl. Kosmich. Fiz., Kiev, N 5, 141.

Vsekhsvyatskij, S. K. 1971, Probl. Kosmich. Fiz., Kiev, N 6, 73.

Vsekhsvyatskij, S. K. 1972, Solnechnaya sistema in Probl. Sovrem. Kosm., Ed. V. A. Ambartsumyan, Nauka, Moscow. 
Vsekhsvyatskij, S. K. 1974, Kosmich. Issledov. na Ukraine, Kiev, N $5,3$. Vsekhsvyatskij, S. K. 1975, Probl. Kosmich. Fiz., Kiev, N 10, 96.

Woolfson, M. M. 1969, Progr. Phys., 32, 135.

\section{Editor's Note}

Professor Vsekhsvyatsky is well known in the western world by the translation from Russian of his major work, "Physical Characteristics of Comets" (NASA TT F-80, Washington, D. C. 1964) which still is one of the major sourcebooks for the absolute magnitudes of comets. Since his recent developments on the eruptive evolution of the solar system are poorly known outside the Russian literature, he had been invited to participate to the Lyon IAU Colloquium in 1976 . He was not allowed to come but he sent a controversial although extremely interesting contribution to this book, that unfortunately had to be drastically edited for length without his help, because of mail and language difficulties. A history of the last 160 years of cometary research, a detailed integration of the amount of cometary and meteoritic matter lost by the solar system, and a philosophical discussion on the inertia of scientific thinking have been cutoff, as less relevant to the purpose of this review. The rest of the text, although shortened to avoid redundancy, remains very close to the original version. A discussion of all ideas including Vsekhsvyatsky's, concerning the origin of comets is given elsewhere in this book. 